\title{
Making the Universe hang together
}

A simple explanation why most of the mass in the Universe is not visible unites questions such as the ionization of intergalactic clouds and the age of the Universe in an arresting way.

THE past few months appear to have brought endless disappointments for those hunting for the dark matter that is supposed to give the Universe the appearence of being almost held together. Every few weeks or so, there seems to have been yet another demonstration that neither the hypothetical particles called axions nor those called photinos will quite fill the bill. Yet the faith of the hunters continues to be sustained not just by hope, but by the orthodox interpretation of observations.

The Universe is indeed expanding less quickly than would be inferred from the distribution of visible matter in galaxies and the stars of which they consist, suggesting quantities of unseen mass up to five times as great as that which can be seen. And the motion of stars and other objects (hydrogen clouds, for example) in the galaxies close enough to be observed in detail is often consistent only with a total mass much greater than that which can be seen.

The puzzle of the missing mass is not, of course, the only puzzle over something missing from the Universe that now keeps astrophysicists awake at nights. There is also the problem of the "missing" neutrinos from the Sun, the twenty-year-old discrepancy between the flux of neutrinos expected from the Sun as by-products of nuclear reactions in the core and the flux measured by neutrino detectors on the Earth. The first measurements, at the Homestake gold mine in the United States, revealed a discrepancy of a factor of three to four between the expected and measured fluxes. That dilemma has, if anything, been sharpened by more recent measurements, as Michael Cherry explained in a News and Views article a few weeks ago (Nature 347, 708; 1990).

But now there is a chance that the two problems may be linked. That seems to be one implication of an article by Denis W. Sciama, the cosmologist who boasts affiliations with the Universities of Oxford and Cambridge as well as with the international institutes of Theoretical Physics and of Advanced Studies at Trieste (Phys. Rev. Lett. 65, 2839; 1990). More accurately, Sciama offers neutrinos with mass as candidate constituents of the missing mass. And these are likely to have arisen only by the spontaneous conversion of ordinary electron neutrinos into the cousin neutrinos associated with the $\mu$ and $\tau$ leptons, the more massive analogues of the electron.
This is not a new idea, as Sciama is careful to acknowledge. That the dark matter may be made of neutrinos with mass has been canvassed on several occasions. Sciama's achievement is that he has found a way of linking together observations of several different kinds with a single and tightly constrained hypothesis about the character of the neutrinos. The theory may be "speculative" as he confesses, but it is also eminently testable. How many astrophysicists can say that of their theories?

The starting point is also unexpected the density of ionized hydrogen in the Galaxy (which can be estimated from the disperson of the signals from galactic pulsars) and the ionization of hydrogen in remote regions of the Universe (where the redshift is large), which are recognizable by the Lyman- $\alpha$ radiation they emit. Why is there so much ionized hydrogen so widely distributed?

The simple answer is that the ionization requires a source of ultraviolet radiation. Hydrogen in a galaxy will be ionized by the ultraviolet radiation from the stars that it contains, but Sciama argues that this is not a sufficiently powerful source, and that the Lyman- $\alpha$ radiation of the distant hydrogen clouds is also "difficult to explain". So what else is there? What, for example, if the dark matter of which the missing mass consists is liable to spontaneous decay, with the emission of ultraviolet photons? The $\mu$ and $\tau$ neutrinos, if sufficiently massive, would fit the bill. It is just a decade since De Rujula and Glashow suggested that it should be possible to recognize particulate missing mass by the homogenous ultraviolet background it would create.

Sciama's objective is to fit his hypothesis that the dark matter consists of neutrinos with such data as there are. His anchor-point is an estimate based on Lyman- $\alpha$ radiation from intergalactic clouds that the flux of ionizing photons must be less than $6 \times 10^{5} \mathrm{~cm}^{-2} \mathrm{~s}^{-1}$. As it happens, the density of neutrinos throughout the Universe is believed to be about $100 \mathrm{~cm}^{-3}$, while the energy required to ionize a hydrogen atom in its own restframe is $13.6 \mathrm{eV}$.

There are a very few straws with which to make bricks. Sciama first supposes that the ionizing photons arise from the conversion of one particle (with mass $m_{1}$ say) into another (with mass $m_{2}$ ). If $m_{1}$ is substantially larger than $m_{2}$, then the energy of the photon is simply $1 / 2 m_{1}$. (Without approximation, it amounts to $\left(m_{1}^{2}-m_{2}^{2}\right) /$ $2 m_{1}$.) This in turn fixes the mass of the more massive of the two neutrinos as having to be greater than $27.2 \mathrm{eV}$. Taking account of a recent failure to observe decay photons from the Coma galactic cluster (with less energy than that required for hydrogen ionization), and allowing for the recession of the cluster calculated from its redshift, he reaches the more stringent limit that $m_{2}>13.9 \mathrm{eV}$.

But that is only a lower limit. How far above it does the true mass lie? Or how to obtain an upper limit for the greater of the two masses? Ingeniously, Sciama links together in two steps almost everything there is in cosmology. First, he obtains a more accurate neutrino density $(115 \pm 2$ $\mathrm{cm}^{-3}$ ) by using the measured intensity of the microwave background and some lore derived from the standard model of particle physics. Then he notes that the ionizing flux will be determined by the proportion of these neutrinos not receding so quickly that the energy of the photons they emit is reduced below the ionization threshold, which brings Hubble's constant (which scales the expansion speed) into the argument.

Two assumptions then make everything hang together. First, Hubble's constant is about $50 \mathrm{~km} \mathrm{~s}^{-1}$ per megaparsec (near the lower limit of the range in which it is constrained by distance measurements) and the lifetime of the more massive neutrino is less than $3 \times 10^{23}$ seconds. Then, the mass of the heavier neutrino $m_{1}$ lies between 27.8 and $30 \mathrm{eV}$, while that of the less massive neutrino (either the electron or the $\tau$ neutrino) is less than $3 \mathrm{eV}$. And, surprising though it may seem, the mass density of neutrinos throughout the Universe turns out to be more than 20 times greater than the estimated density of baryonic matter. Plainly, there is enough missing mass to satisfy everybody. For what it is worth, the age of the Universe also tumbles out at 12,000 million years.

What does all this mean? It would be easy but mistaken to dismiss the argument as a tissue of speculation. In reality, Sciama has a reputation among cosmologists for believing that the numbers matter, even if there too few of them for comfort. What he has done is to construct a picture of the Universe that has the merit of being testable. It will be interesting to see what they make of it at the symposium on cosmology and particle physics arranged for next week at the University of Sussex.

John Maddox 